\title{
Perfil epidemiológico e clínico de pacientes portadores de artrite reu- matóide em um hospital escola de medicina em Goiânia, Goiás, Brasil*
}

\section{Epidemiological and clinical profile of patients with rheumatoid arthritis in a medical academic hospital in Goiania, Goias, Brazil}

Andrey Evangelista Vaz ${ }^{1}$, Wilmar Agostinho Faria Júnior ${ }^{1}$, Cristina Ferreira e S. Lazarski², Humberto Franco do Carmo $^{2}$, Hermínio Maurício da Rocha Sobrinho ${ }^{3}$

\begin{abstract}
RESUMO
Objetivo: realizar uma análise das principais características clínico-demográficas de pacientes com artrite reumatóide (AR), em acompanhamento ambulatorial, em um Hospital Escola de Medicina em Goiânia-GO. Metodologia: Trata-se de uma análise epidemiológica, retrospectiva, de um conjunto de pacientes com artrite reumatóide acompanhados por um hospital universitário da região centro-oeste do Brasil. Foram analisados 500 prontuários médicos de pacientes atendidos no ambulatório de reumatologia no período de 2011 a 2012. Os dados dos prontuários foram transcritos para um documento de protocolo padronizado e validado por um médico reumatologista. Resultados: do total de 500 pacientes analisados, $43 \%$ apresentavam doenças reumáticas sistêmicas, $36 \%$ doenças osteoarticulares, $10 \%$ espondiloartrites e $11 \%$ outras doenças. Deste total, $5 \%$ eram pacientes portadores de AR. A relação entre o sexo feminino/masculino foi de 4:1, a média de idade dos pacientes foi de $50 \pm 13,3$ anos, com tempo médio de doença de 9,7 $\pm 5,6$ anos. A história familiar de AR foi presente em $16 \%$ dos pacientes, $42 \%$ apresentavam alterações radiológicas na primeira consulta e $69 \%$ apresentavam comorbidades. Os principais sinais e sintomas referidos pelos pacientes foram dor articular (89\%), rigidez articular matinal $(73 \%)$ e edema articular $(73 \%)$. As manifestações extra-articulares ocorreram em $21 \%$, o fator reumatóide foi positivo em $68 \%$ dos pacientes e $26 \%$ do total de pacientes com AR eram positivos para autoanticorpos antinucleares. Apenas $10 \%$ dos pacientes realizavam tratamento fisioterapêutico e o fármaco mais utilizado como tratamento medicamento da AR foi o Metotrexato. Conclusão: este estudo proporcionou uma visão parcial do perfil epidemiológico de pacientes com AR de um hospital universitário em Goiânia-GO. Observou-se uma pequena utilização de marcadores diagnósticos da doença. Diante disso, propomos uma devolutiva para o ambulatório de reumatologia na tentativa de melhorar a qualidade e disponibilidade das informações médicas nos prontuários deste ambulatório do hospital escola de medicina da PUC Goiás.
\end{abstract}

Palavras-chave: Artrite Reumatóide. Hospitais-Escola. Perfil Epidemiológico. Goiânia-GO.

* O artigo originou-se de um trabalho de conclusão do curso de Medicina da Pontifícia Universidade Católica de Goiás, Goiânia, 2012 (Artigo Original).

1. Acadêmicos do último ano do curso de Medicina da PUC Goiás

2. Médicos reumatologistas do Hospital Escola Santa Casa de Misericórdia de Goiânia-GO.

3. Professor Assistente de Imunologia do Departamento de Medicina da PUC Goiás.
Correspondência: Pontificia Universidade Católica de Goiás Departamento de Medicina Av. Universitária, n.ำ 1069, Setor Universitário CEP 74.605-010 - Goiânia - Goiás. herminio.sobrinho@gmail.com

Artigo recebido em 17/12/2012 Aprovado para publicação em 23/04/2013 


\section{Introdução}

A artrite reumatóide (AR) é uma doença inflamatória crônica sistêmica, autoimune, que acomete especialmente os tecidos sinoviais, podendo haver envolvimento extra-articular. Está usualmente relacionada ao desenvolvimento de deformidades e incapacitação, gerando diminuição da qualidade de vida e morte prematura. ${ }^{1}$

A prevalência da AR é de cerca de 3:1 mulheres em relação aos homens e acomete atualmente cerca de $1 \%$ da população mundial, podendo variar entre grupos populacionais, sendo sua ocorrência observada em todos os grupos étnicos. A doença costuma se apresentar, sobretudo, entre a $4^{\mathrm{a}}$ e a $6^{\mathrm{a}}$ década de vida, acometendo três vezes mais mulheres com idade inferior a 45 anos em relação aos homens. Esta diferença diminui com o avanço da idade e tende a se igualar após os 65 anos. ${ }^{2,3}$ Estudo multicêntrico brasileiro em amostras populacionais das macrorregiões do país - norte, nordeste, centro-oeste e sul - encontrou prevalência de ate $1 \%$ da população adulta, que corresponderia a uma estimativa de 1.300 .000 pessoas acometidas. ${ }^{4}$

A AR acomete indivíduos em idade produtiva no trabalho e pode determinar importante limitação na capacidade funcional e perda de capacidade laboral, assim, custos indiretos relacionados a esse contexto devem ser incorporados as análises de farmacoeconomia. $^{5}$

As características demográficas e clínicas da AR são variáveis de acordo com a população acometida. A maior parte das informações disponíveis provem da Europa e dos Estados Unidos. São poucos os estudos realizados na população brasileira e principalmente na região centro-oeste. ${ }^{6,7}$

O diagnóstico da AR é estabelecido considerando-se achados clínicos e exames complementares. Nenhum teste isolado, seja laboratorial, de imagem ou histopatológico confirma o diagnóstico. ${ }^{8}$

O ambulatório de reumatologia do Hospital Escola Santa Casa de Misericórdia de Goiânia-GO (SCMG-GO) recebe pacientes com doenças reumáticas encaminhados de várias regiões do estado de Goiás para realização do tratamento e acompanhamento da doença, sendo conveniado com o Sistema Único de Saúde (SUS). Os encaminhamentos são realizados pela central de regulação do SUS do Estado de Goiás. Este ambulatório é operacionalizado por quatro (4) médicos reumatologistas contratados pela instituição de ensino superior (PUC Goiás), os quais, durante as consultas médicas, supervisionam e acompanham os acadêmicos do curso de medicina da PUC Goiás a partir do $8^{\circ}$ módulo até o $12^{\circ}$ módulo. Esta unidade de reumatologia oferece quatro (4) ambulatórios por semana, com duração média de 3 horas cada consulta, atendendo, no total, cerca de 20 pacientes reumáticos por semana.

Um estudo desta natureza ainda não havia sido realizado na região centro-oeste. Diante disso, esta pesquisa propôs-se a coletar informações clínico-demográficas, dos prontuários médicos, de pacientes portadores de artrite reumatóide atendidos no período de 2011 a 2012, no ambulatório de reumatologia deste hospital universitário em Goiás, para verificar a possibilidade de se caracterizar um perfil epidemiológico desta doença reumática autoimune em Goiânia-GO.

\section{Material e Métodos}

O presente estudo é resultado do trabalho de conclusão do curso de medicina de uma dupla de acadêmicos do último ano do curso de medicina da PUC Goiás em Goiânia-GO. Este foi aprovado pelo Comitê de Ética em Pesquisa da Santa Casa de Misericórdia de Goiânia-GO pelo protocolo $n^{\circ}$ 003/2012, sendo posteriormente realizado no ambulatório de reumatologia deste hospital universitário.

Foi realizado um levantamento de quinhentos $(\mathrm{n}=500)$ prontuários médicos, no período de Março a Outubro de 2012, de pacientes com doenças reumáticas atendidos no período de Fevereiro de 2011 a Outubro de 2012 neste ambulatório médico.

Trata-se de uma análise epidemiológica, retrospectiva, de um conjunto de pacientes com artrite reumatóide acompanhados por um hospital universitário da região centro-oeste do Brasil. Embora o número de pacientes incluídos seja pequeno, corresponde a uma região do país carente em estudos dessa natureza. Foram analisados prontuários de vinte e cinco $(n=25)$ pacientes com diagnóstico AR, segundo critérios de classificação diagnóstica do Colégio Americano de Reumatologia, atendidos nesta unidade de saúde. Todos os pacientes participantes do estudo tinham comparecido em mais de uma consulta ambulatorial no período do estudo. ${ }^{9,10}$

Realizou-se uma extensa e minuciosa avaliação de diversos aspectos clínico-demográficos associados aos pacientes portadores de artrite reumatóide para caracterização do perfil epidemiológico destes indivíduos atendidos no hospital escola de medicina da PUC Goiás, em Goiânia, Goiás. 


\section{Pacientes}

A coleta de dados dos prontuários médicos ocorreu mediante o preenchimento de um instrumento de coleta de dados validado por um médico reumatologista, no qual eram transcritos os seguintes dados clínicodemográficos: idade, sexo, tempo de diagnóstico da doença, historia familiar de AR, triagem do paciente, critérios de diagnóstico da doença, exames para diagnóstico da doença, alterações radiológicas, deformidades articulares, envolvimento extra-articular, tratamento, co-morbidades e estado geral da doença (com base nos critérios de capacidade funcional do Colégio Americano de Reumatologia: leve ou grau I, moderada ou grau II e grave ou grau III, IV). ${ }^{10}$

Foram incluídos no estudo dezenove $(n=19)$ pacientes ambulatoriais do Sistema Único de Saúde (SUS), com diagnóstico de AR, que preencheram no mínimo $70 \%$ das questões do instrumento de coleta de dados utilizado. Excluiu-se deste estudo seis $(n=6)$ pacientes ambulatoriais que não preencheram o mínimo de $70 \%$ das questões do instrumento de coleta de dados.

\section{Análise estatística dos dados}

As variáveis epidemiológicas e clínicas foram analisadas de forma descritiva, por meio de valores percentuais, média, desvio padrão, gráficos, utilizando-se o software Microsoft Office Excel versão 2007.

\section{Resultados}

Observou-se que as principais doenças reumáticas acompanhadas no ambulatório de reumatologia deste hospital universitário foram as doenças reumáticas sistêmicas $(n=215)$, outras doenças osteoarticulares $(n=175)$, espondiloartrites $(n=48)$ e doenças não reumatológicas $(n=52)$, conforme gráfico 1 . Convém ressaltar que considerou-se, como espondiloartrite, neste estudo, apenas as doenças inflamatórias da coluna vertebral (esqueleto axial) com envolvimento inflamatório de ligamentos e tendões nas interfaces entre o osso e a cartilagem articular (entesites). No grupo das doenças não reumatológicas verificou-se a presença de infecções (artrite séptica), hemocromatose, mieloma múltiplo, sarcoidose e angioedema.

Foi observado, neste trabalho, uma maior prevalência da AR no sexo feminino, conforme Tabela 1, com 15 pacientes do gênero feminino e 4 do gênero masculino, chegando-se a uma relação sexo feminino/masculino de 4:1. A média de idade dos pacientes com AR foi de 50 anos, com desvio padrão de 13,3. Os pacientes apresentavam, em média, 10 anos de evolução da doença, com desvio padrão de 5,6. Apenas três pacientes apresentavam história familiar de AR, correspondendo a $16 \%$ do total analisado. No entanto, 13 pacientes (68\%), apresentavam co-morbidades (hipertensão arterial sistêmica, diabetes mellitus, hipotireoidismo, osteoartrose, osteoporose, etc.).

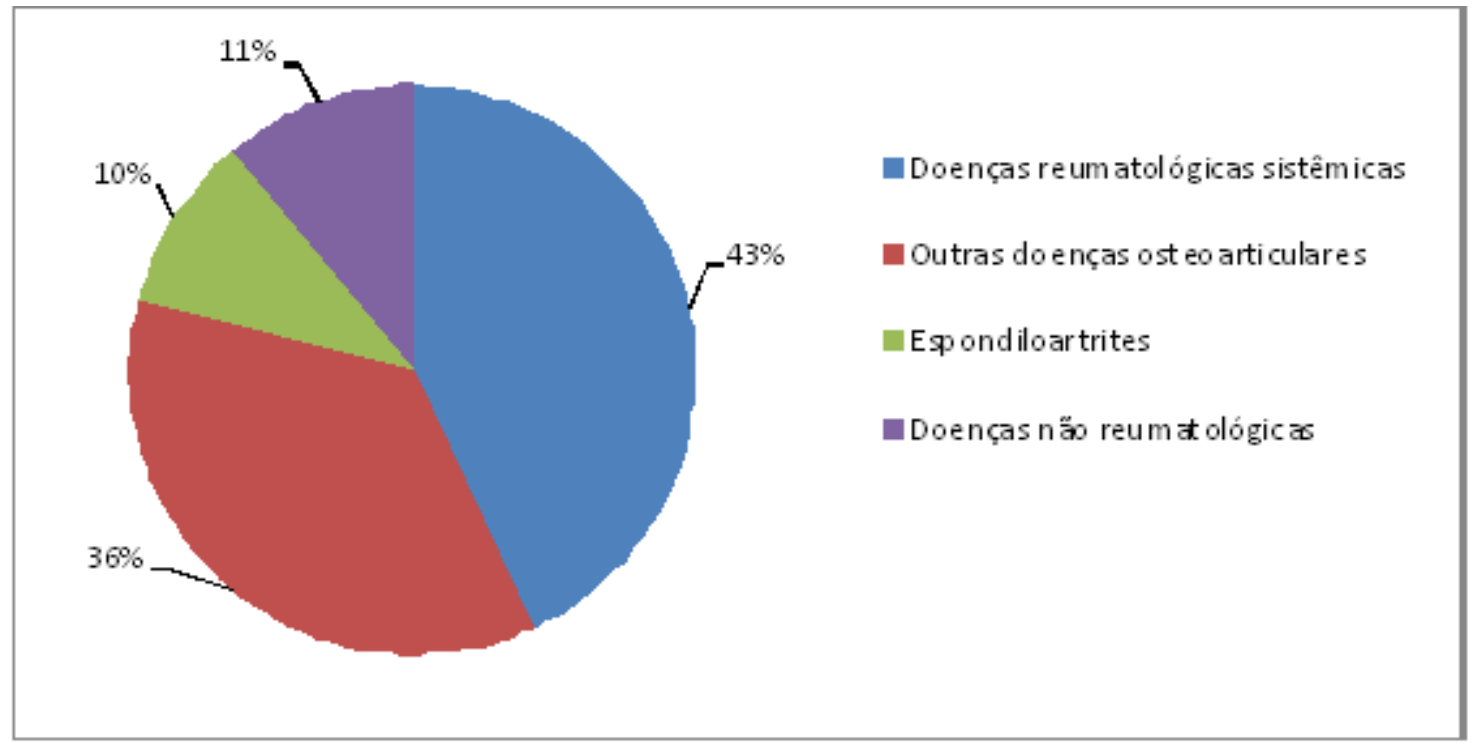

Gráfico 1. Grupo de doenças levantadas nos prontuários médicos do setor de reumatologia do hospital escola Santa Casa de Misericórdia de Goiânia no período de 2011 a $2012(n=500)$. 


\section{Tabela 1}

Características clínicas e demográficas dos pacientes portadores de Artrite Reumatóide (AR) atendidos no ambulatório de reumatologia da SCMG no período de 2011a 2012, n = 19 .

\begin{tabular}{ll}
\hline Características clínicas e demográficas & Valores \\
\hline Sexo feminino/masculino & $15(79 \%) / 4(21 \%)$ \\
Raça & $\mathrm{NI}^{\mathrm{a}}$ \\
Média de idade em anos (Md + DP) & $50,0 \pm 13,3$ \\
Tempo de doenças em anos (Md + DP) & $9,7 \pm 5,6$ \\
Historia Familiar de AR & $3,0(16 \%)$ \\
Presença de co-morbidades & $13,0(68 \%)$ \\
Presença de deformidades articulares na $1^{\mathrm{a}}$ consulta & $8,0(42 \%)$ \\
Fator reumatóide sérico positivo & $13,0(68 \%)$ \\
Pesquisa de autoanticorpos séricos positivos (FAN) & $4,0(21 \%)$ \\
Impressão clínica geral & Leve-47\% \\
& Moderada - 31\% \\
\end{tabular}

$\mathrm{Nl}^{\mathrm{a}}$ Não informada (prontuários médicos). Ambulatório de Reumatologia SCMG, Goiânia-GO.

Dos 19 pacientes com AR analisados, 8 pacientes (42\%) apresentavam deformidades articulares já na $1^{\mathrm{a}}$ consulta, destes $13(68 \%)$ apresentavam apenas o anticorpo sérico fator reumatóide (FR) positivo. A pesquisa de autoanticorpos séricos antinucleares positivos (FAN) estava presente em apenas 4 pacientes (21\%). A impressão clínica geral dos pacientes com $\mathrm{AR}$ atendidos no ambulatório de reumatologia da SCMG-GO foi de $47 \%$ casos leves, $31 \%$ de casos moderados, $22 \%$ de casos graves de Artrite Reumatóide (critérios baseados na avaliação física, achados laboratoriais, exames radiológicos e medicação utilizada). Não obtivemos dados a respeito da raça e profissão dos pacientes, assim como de padrões celulares de fluorescência nos exames de imunofluorescência indireta (IFI) para detecção de autoanticorpos séricos, pois tais dados não foram informados nos prontuários médicos.

Quanto ao tempo de diagnóstico da AR, dos 19 pacientes analisados, $26 \%$ tinham menos do que 5 anos de doença, $42 \%$ entre 5 e 10 anos, $5.5 \%$ entre 10 e 15 anos, $21 \%$ entre 15 e 20 anos e $5.5 \%$ apresentavam mais de 20 anos de diagnóstico de AR (Gráfico 2).

Apenas $16 \%$ dos pacientes analisados apresentavam história familiar de AR (gráfico 3). Cerca de $63 \%$ dos pacientes com AR apresentavam exame clínico, exames laboratoriais, e exames radiológicos como subsídios para diagnosticar a AR, enquanto $27 \%$ apresentavam apenas exames clinico e laboratoriais (gráfico 4).

Em relação ao processo de encaminhamento dos pacientes com AR para a SCMG-GO, observamos, que do total de 19 pacientes com AR, 63\% foram encaminhados, ao ambulatório deste hospital universitário, por médicos reumatologistas, $11 \%$ por ortopedistas e $26 \%$ por clínico geral (gráfico 5).

Sobre os exames laboratoriais positivos, observamos, que 13 pacientes (68\%) apresentavam apenas FR sérico positivo, 3 (16\%) com FR e FAN positivos, 1 paciente com apenas o FAN positivo e 2 pacientes $(11 \%)$ foram Soro Negativos para FR e autoanticorpos antinucleares (FAN), conforme gráfico 6 . Do total de pacientes com AR estudados, 8 (42\%) apresentavam alterações radiológicas (descalcifica- 


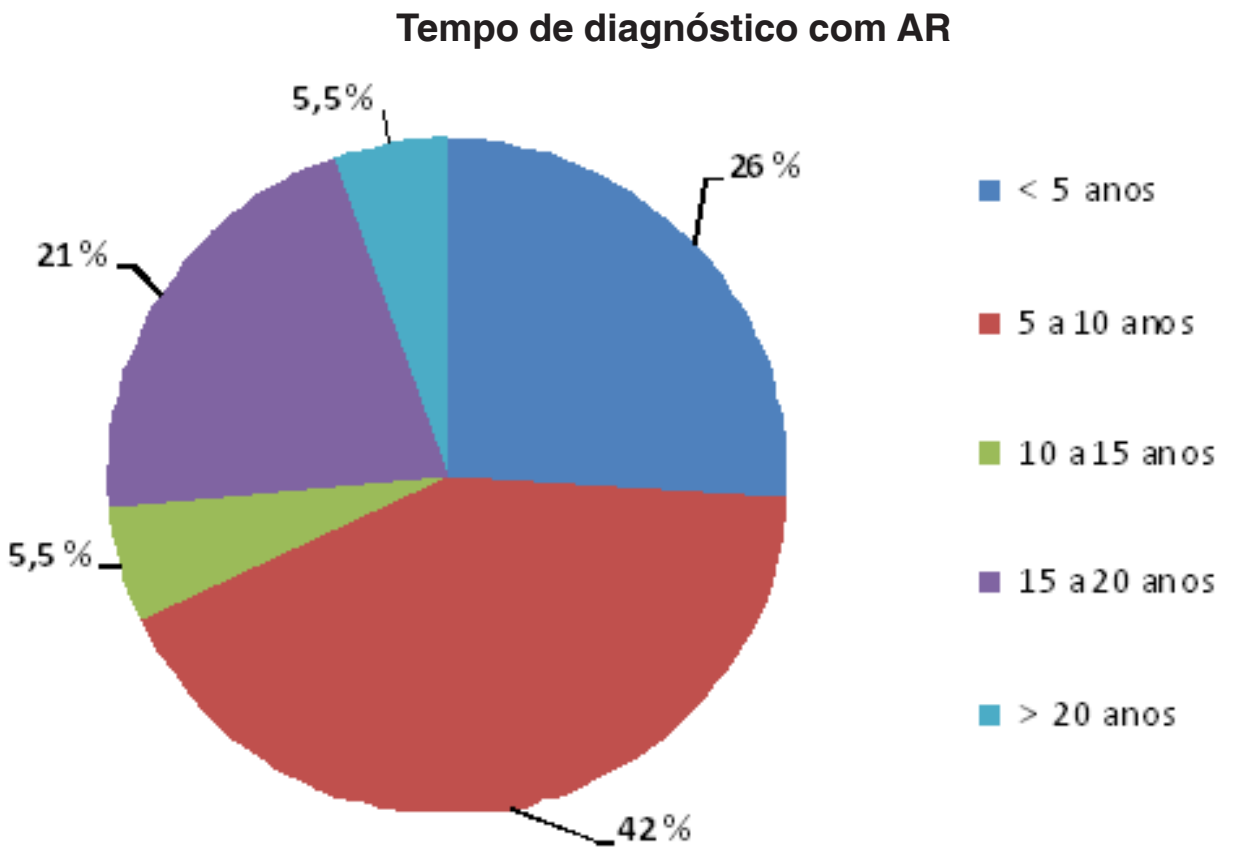

Gráfico 2. Tempo de diagnóstico da doença em anos, $n=19$.

\section{Familiar com AR}

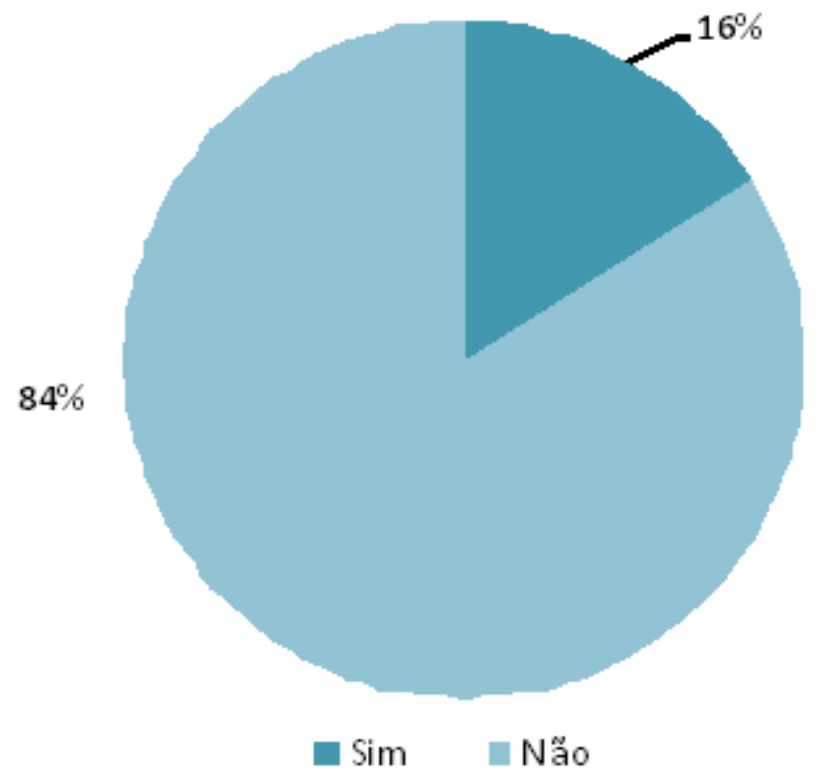

Gráfico 3. Presença de História Familiar de Artrite Reumatóide $(n=19)$

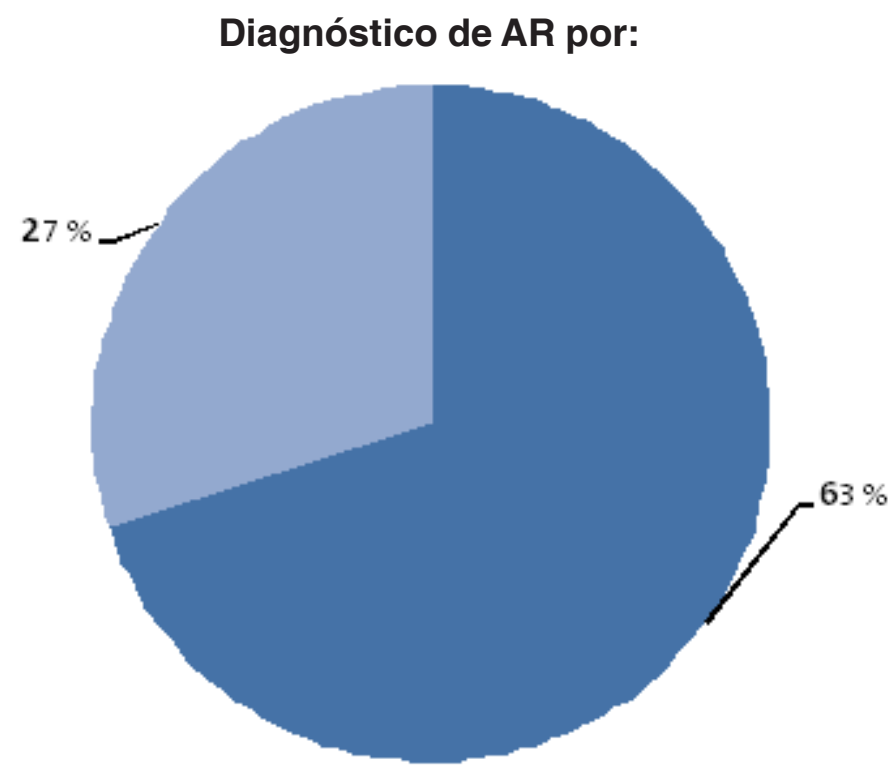

Exame Clínico / Laboratoriais / Radiológico

Exame Clínico / Laboratorial 


\section{Triagem para SCMG-GO para tratamento da AR}

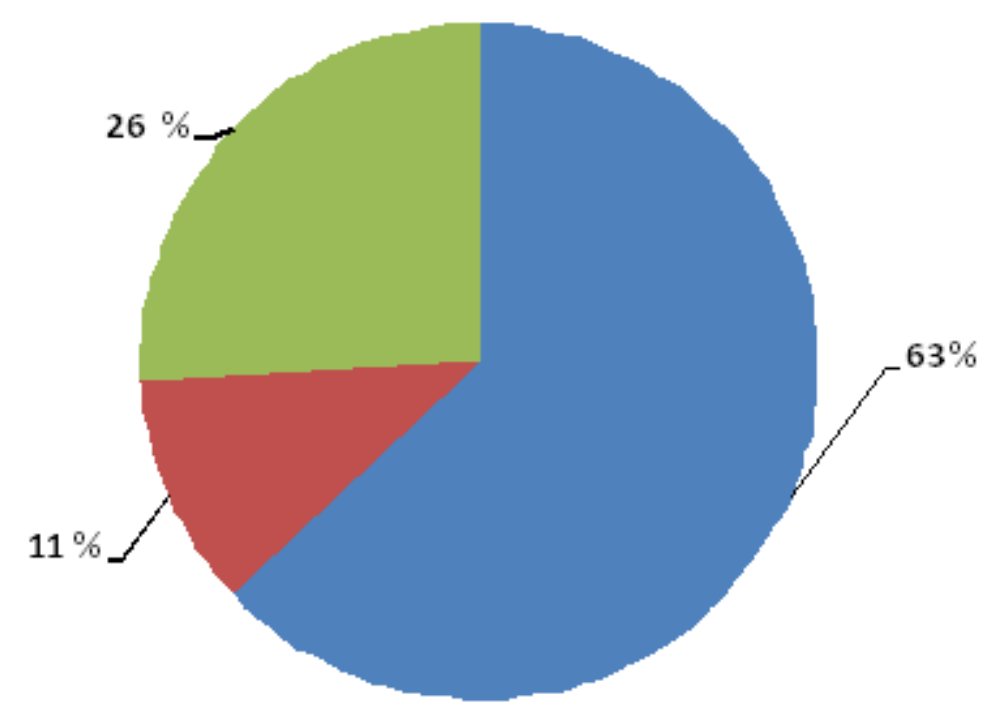

Reumatologista Ortopedista Clínico Geral

Gráfico 5. Especialidade médica responsável pelo encaminhamento dos pacientes ao ambulatório de reumatologia SCMG-GO.

\section{Exames Laboratoriais Positivos}

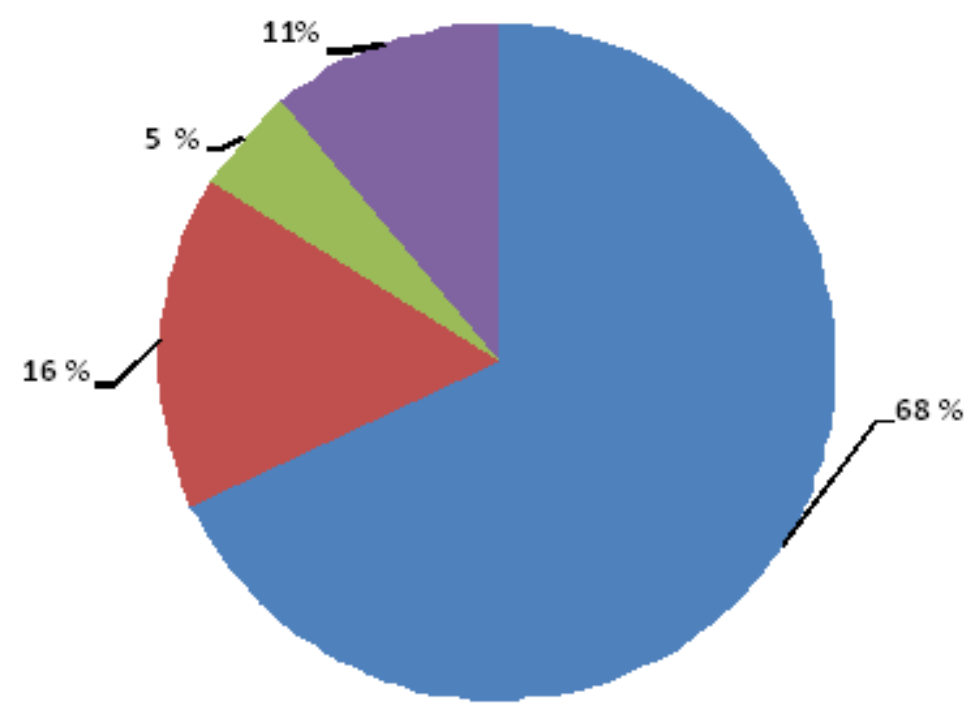

IFR $\square$ FR e FAN $\square$ FAN $\square$ AR Soro Negativa

Gráfico 6. Pesquisa de autoanticorpos séricos em pacientes com AR do ambulatório de reumatologia da SCMG-GO, (n=19). 
ções em mãos e punhos, erosões ósseas, deformidades articulares em mãos, pés e cotovelos), conforme gráfico 7.

Verificou-se à presença de alterações articulares em quase a metade do número de pacientes portadores de AR do ambulatório de reumatologia da SCMG-GO. Observou-se deformidades nas articulações metacarpofalangeanas, interfalangeanas proximais, metatarsofalangeanas, punhos, joelhos, cotovelos e ombros. Encontramos 8 pacientes (42\%) com alterações articulares já na primeira consulta (descalcificações em mãos e punhos, aumento de volume das articulações interfalangeanas proximais, dedos em fuso, dedos em pescoço de cisne), como mostra o gráfico 8.

Quanto ao tratamento, dos 19 pacientes analisados, apenas $15 \%$ realizavam tratamento medicamentoso e fisioterapêutico. Enquanto $85 \%$ destes pacientes realizavam apenas o tratamento medicamentoso (Gráfico 8).

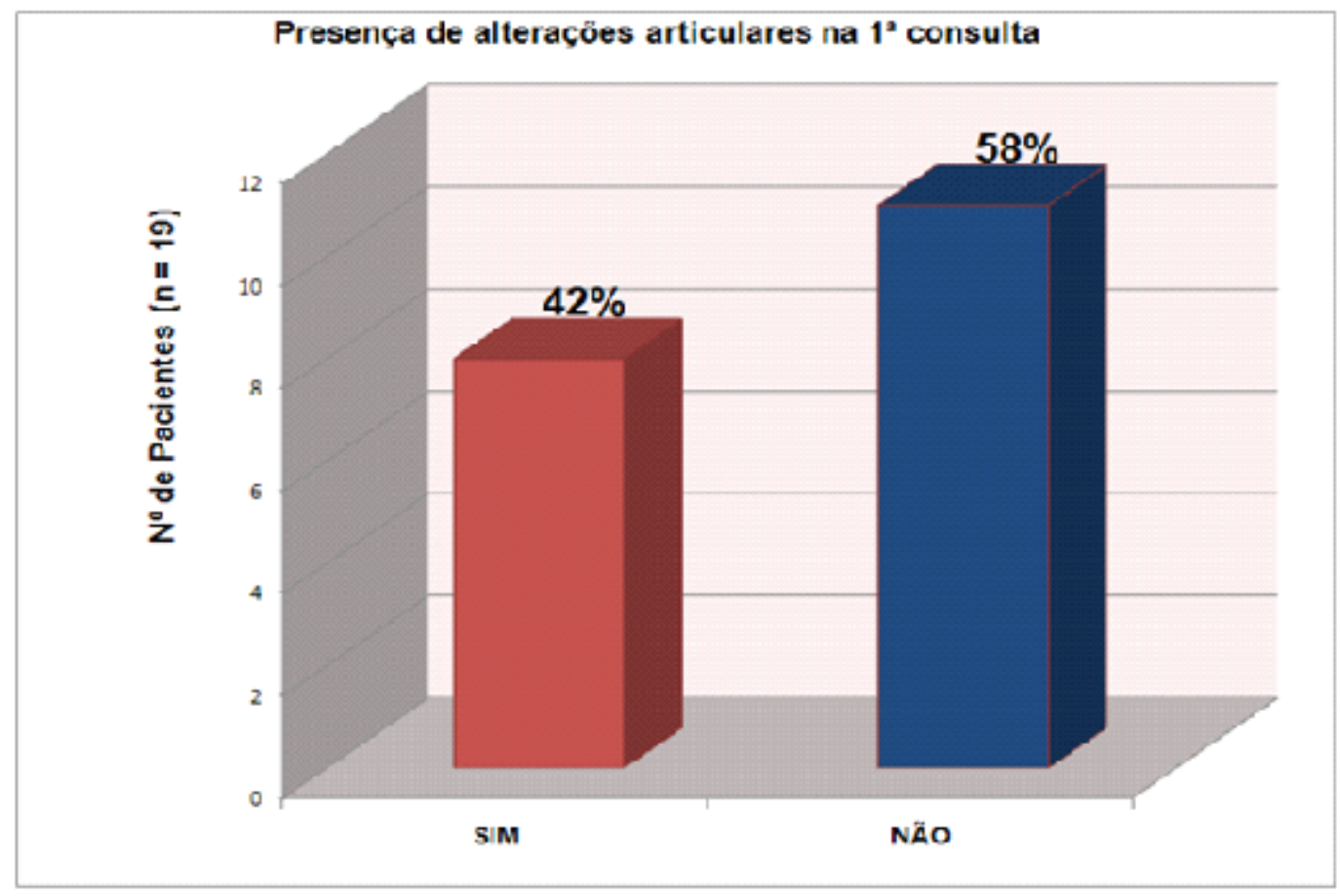

Gráfico 7. Presença de alterações articulares na $1^{\text {a }}$ consulta no ambulatório de reumatologia SCMG-GO.

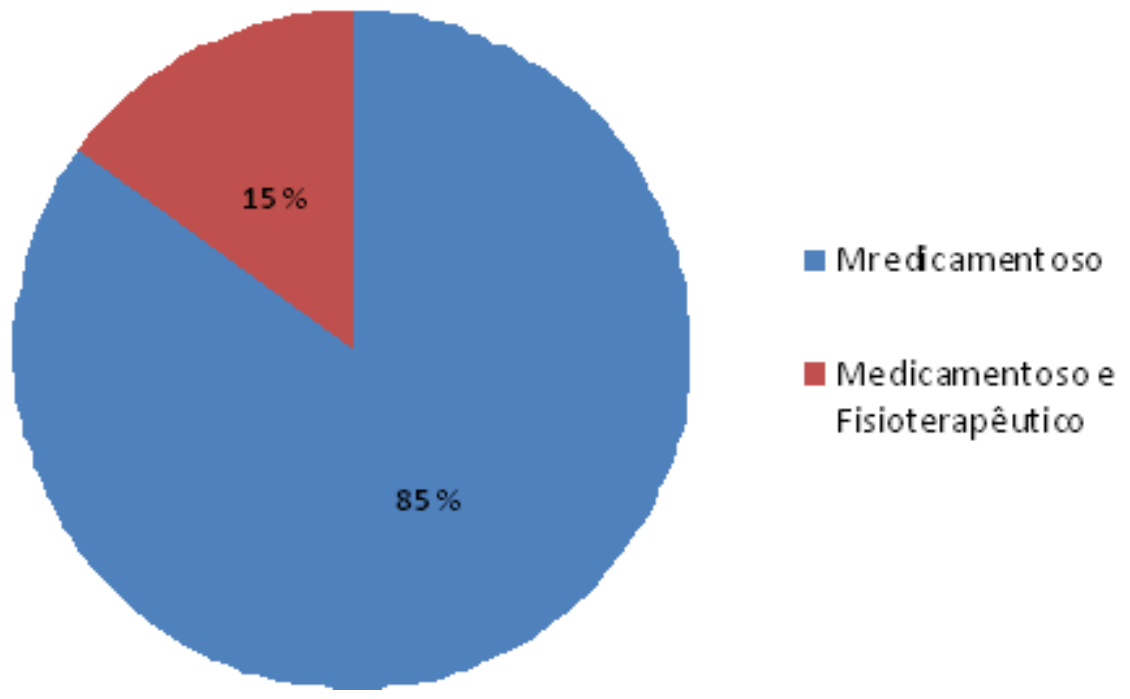

Gráfico 8. Tratamento para AR realizado pelos pacientes do ambulatório de reumatologia da SCMG-GO (2011 - 2012). 
Quanto aos medicamentos utilizados pelos pacientes com AR de nosso ambulatório, 17 pacientes estavam em uso de corticóides, 14 pacientes em uso de AINEs, 15 usando metotrexate, 10 usando cloroquina, e apenas 1 paciente em uso de medicação imunobiológica, entre outras medicações usadas como é mostrado no gráfico 9.
Observamos que $69 \%$ dos pacientes com AR analisados apresentam co-morbidades, conforme gráfico 10. As principais co-morbidades encontradas foram Hipertensão Arterial Sistêmica, Diabetes Mellitus, Hipotireoidismo, Osteoartrose e Osteoporose.

Em relação a presença de lesão extra-articular, observou-se que $79 \%$ de nossos pacientes não

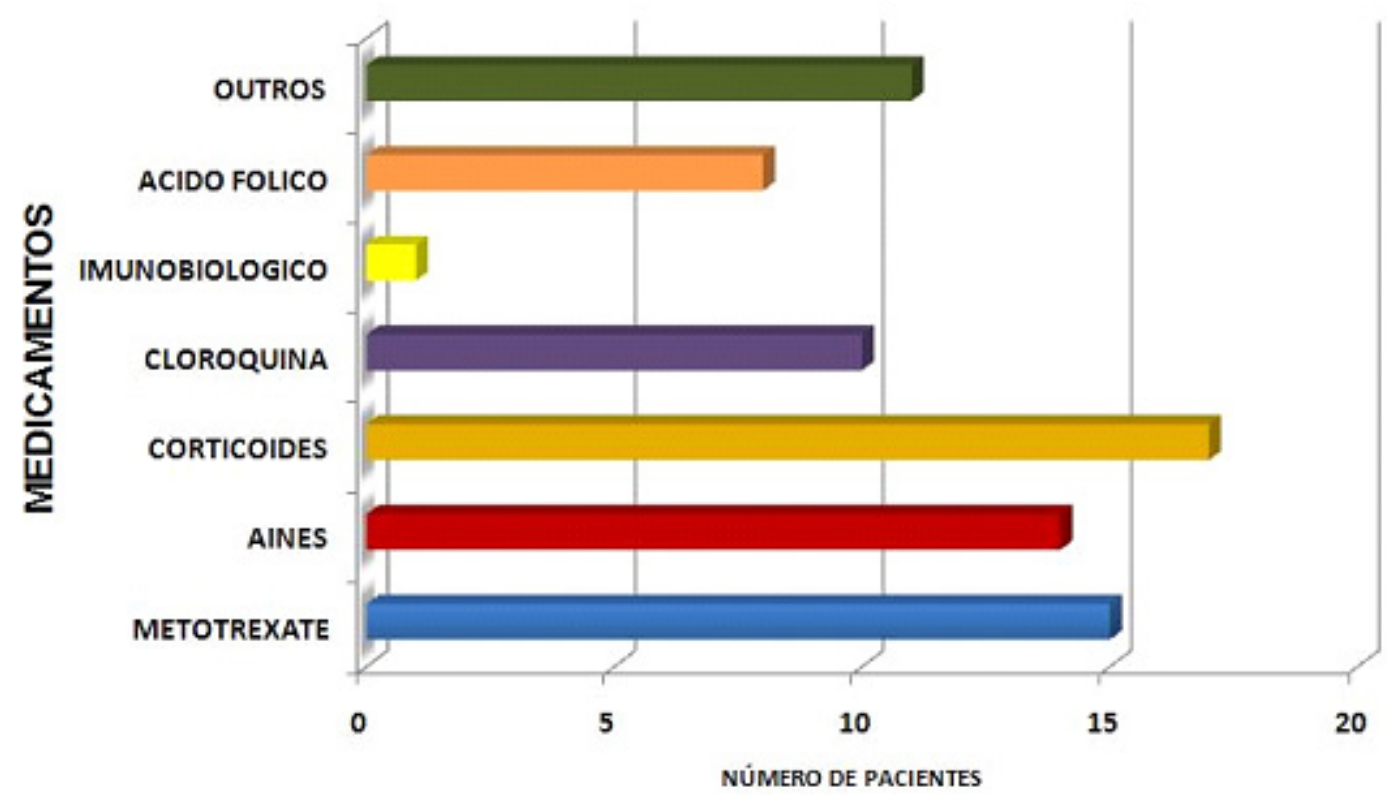

- Outros: Leflunomida, Tramadol, Colchicina, Sulfassalazina, Ciclobenzaprina, Indometacina.

- Imunobiológico utilizado pelo paciente: Adalimumabe.

Gráfico 9. Medicações utilizadas pelos pacientes com AR do ambulatório de reumatologia da SCMG $(n=19)$.

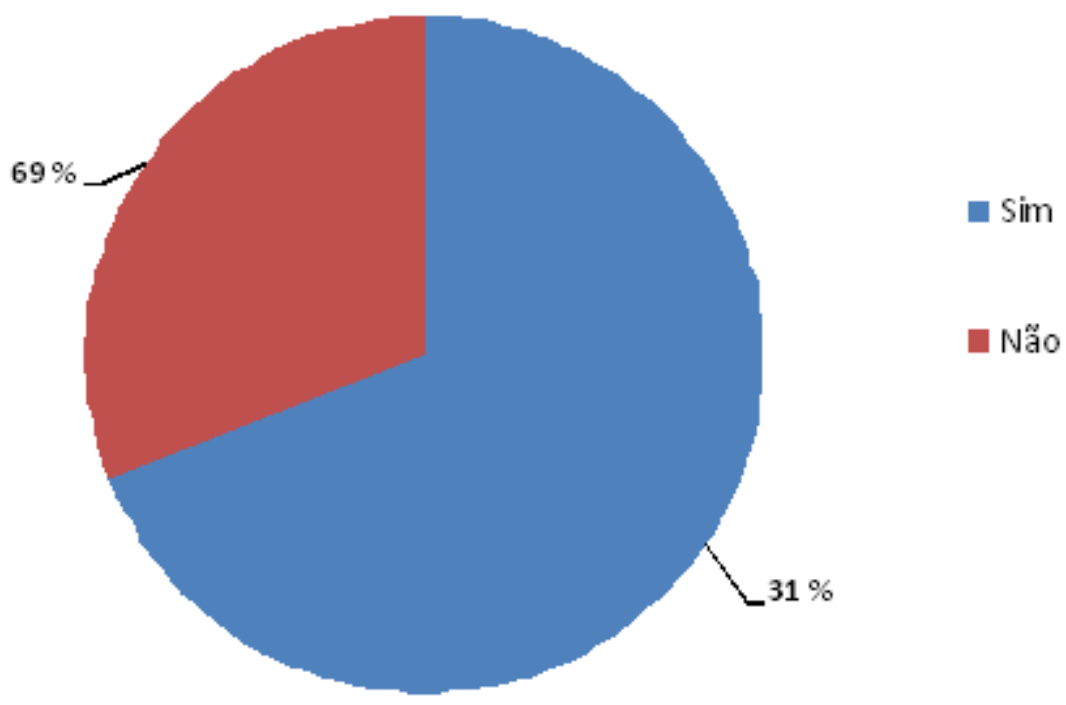

Gráfico 10. Presença de co-morbidades nos pacientes com AR do ambulatório de reumatologia SCMG-GO (n=19). 
apresenta qualquer lesão extra-articular, conforme gráfico 11. A tabela 2 traz dados sobre as manifestações extra-articulares presentes em $21 \%$ dos pacientes com AR.

\section{Presença de lesão extra-articular}

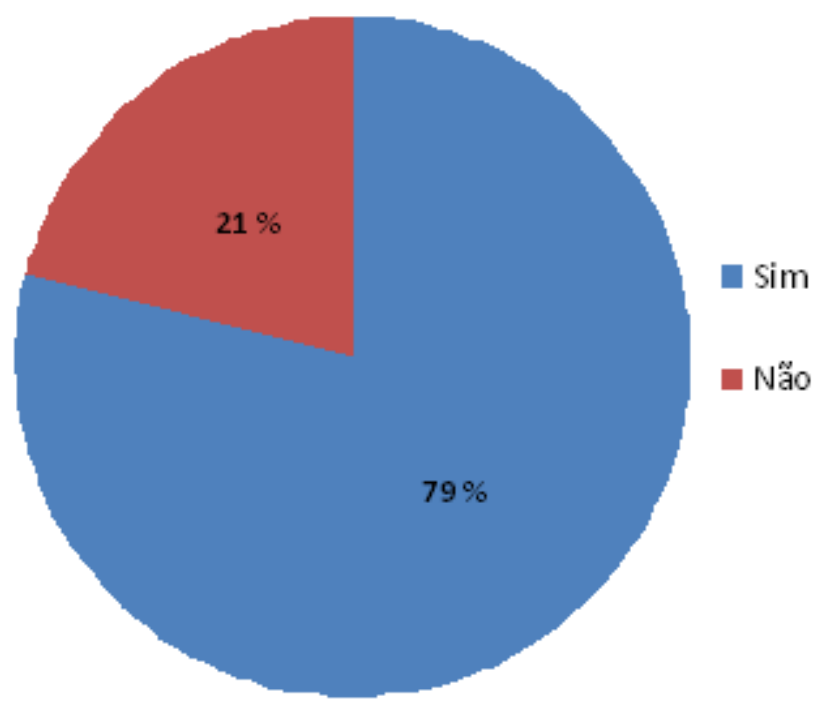

Gráfico 11. Presença de lesão extra-articular nos pacientes com AR do ambulatório de reumatologia SCMG $(n=19)$

\section{Tabela 2}

Manifestações extra-articulares de pacientes com Artrite Reumatóide (AR) atendidos no ambulatório de reumatologia da SCMG no período de 2011a 2012, $\mathrm{n}=19$.

\section{Manifestações clínicas extra-articulares}

Nódulos subcutâneos

$21 \%$

Acometimento ocular

$10 \%$

Neuropatia

$10 \%$

Vasculite

$5 \%$

Ambulatório de reumatologia da Santa Casa de Misericórdia de Goiânia-GO.

Apenas 2 pacientes, dos 19 analisados, haviam freqüentado programas de reabilitação. A grande maioria dos pacientes analisados, 17 pacientes (90\%), não freqüentavam tais programas (gráfico 12).

O número de consultas, dos pacientes com AR $(\mathrm{n}=19)$, no ambulatório de reumatologia da SCMG por

\section{Frequentou programas de reabilitação}

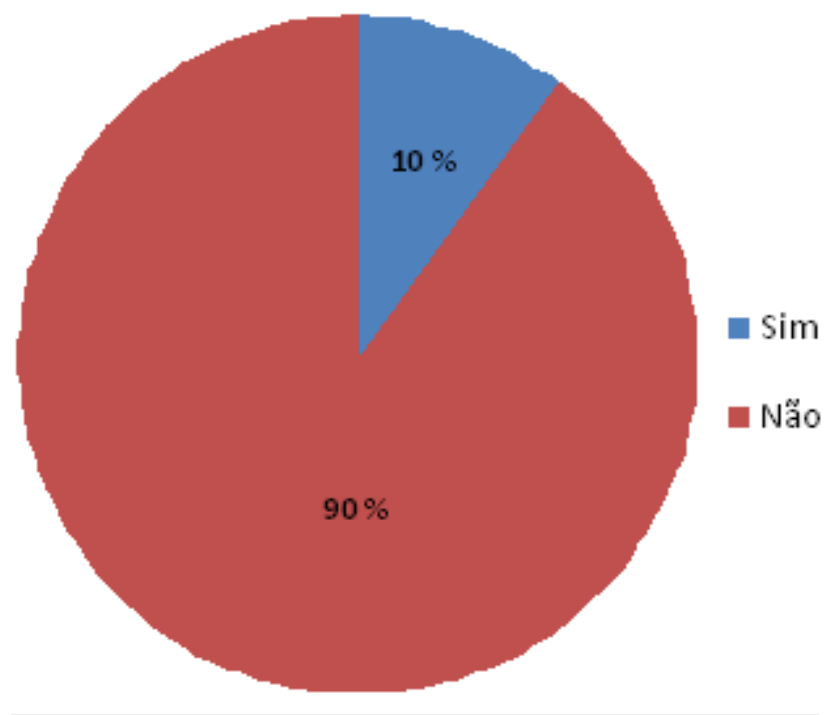

Gráfico 12. Pacientes que freqüentaram Programas de Reabilitação para melhorar qualidade de vida do paciente com AR $(n=19)$.

ano foi, conforme gráfico abaixo, $5 \%$ dos pacientes com uma consulta ao ano, $37 \%$ com duas consultas ao ano, $16 \%$ com três consultas ao ano, $37 \%$ com quatro consultas ao ano e $5 \%$ com mais de 4 consultas ao ano.

\section{№ de consultas no Reumatologista / ano}

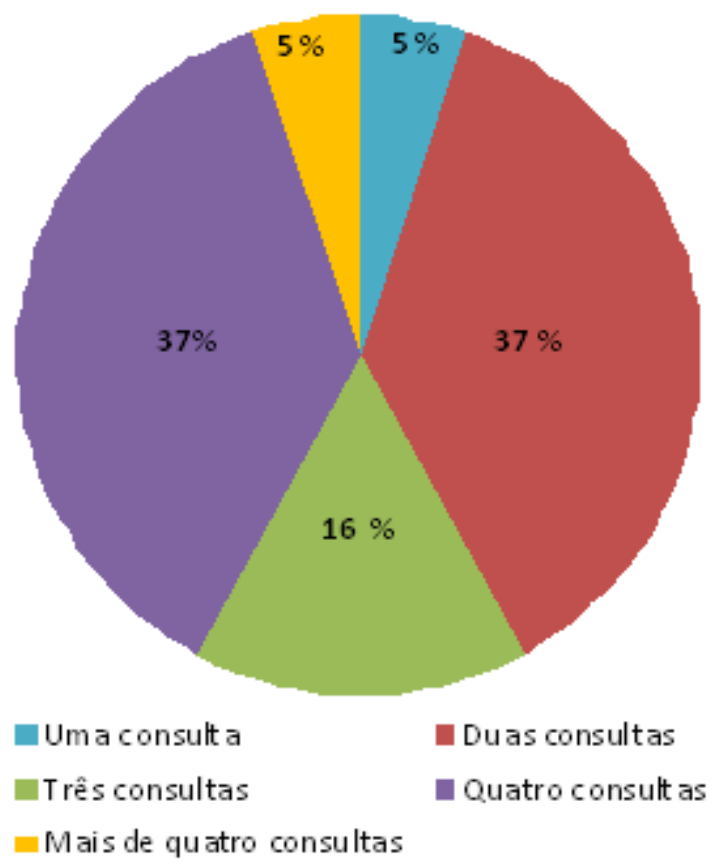

Gráfico 13. Número de consultas realizadas pelos pacientes com AR do ambulatório de reumatologia. 
A respeito dos sinais e sintomas da AR referidos pelos pacientes que participaram deste estudo, observou-se que 14 pacientes $(73 \%)$ referiam rigidez articular matinal, 17 (89\%) pacientes referiam dor articular, 14 pacientes $(73 \%)$ referiam edema articular e $3(15 \%)$ pacientes referiam nódulos subcutâneos, ressaltando-se que cada paciente, normalmente, referia mais de um sinal/sintoma (gráfico 14).

\section{Discussão}

A partir da análise deste estudo clínico-epidemiológico observou-se uma relação de sexo feminino/masculino de 4:1, sendo $79 \%$ dos pacientes com AR do gênero feminino e a idade média dos pacientes foi de $50 \pm 13,3$ anos, maioria entre a quarta e quinta década de vida, que condiz com a estatística mundial e do país, que afeta mulheres duas a três vezes mais do que homens e sua prevalência aumenta com a idade. ${ }^{3,6}$

Quanto ao tempo médio de doença dos pacientes, igual a 9,7 $\pm 5,6$ anos, notamos a proximidade deste valor com os da literatura do país que demonstra a história de início de doença há menos de 10 anos. ${ }^{6,7}$

Nesta pesquisa verificou-se que $16 \%$ dos pacientes apresentavam história familiar de Artrite Reumatóide, dado que corrobora para a presença do componente genético significativo tanto do risco de desenvolvimento quanto para a gravidade da doença. Por conseguinte, há relatos de certas populações em que a artrite reumatóide é pouco frequiente e outras em que ela é mais comum. ${ }^{1,2,3}$

Sobre as manifestações clínicas, foi observado que quase a metade dos pacientes apresentava alterações articulares (radiológicas) já na primeira consulta. Deste total, $40 \%$ deles evoluíram com piora radiológica articular, havendo uma relação direta entre o tempo de diagnóstico e o comprometimento articular, ou seja, quanto maior o tempo de doença pior a evolução. Além disso, as manifestações extra-articulares ocorreram em $21 \%$ dos pacientes, o que pode estar relacionado com a produção e atividade do autoanticorpo fator reumatóide em tecidos extra-articulares, produção de citocinas pró-inflamatórias por linfócitos autorreativos e deposição de imunocomplexos teciduais. As manifestações clínicas encontradas foram: nódulos subcutâneos, envolvimento pulmonar,

\section{Sinais e Sintomas referidos}

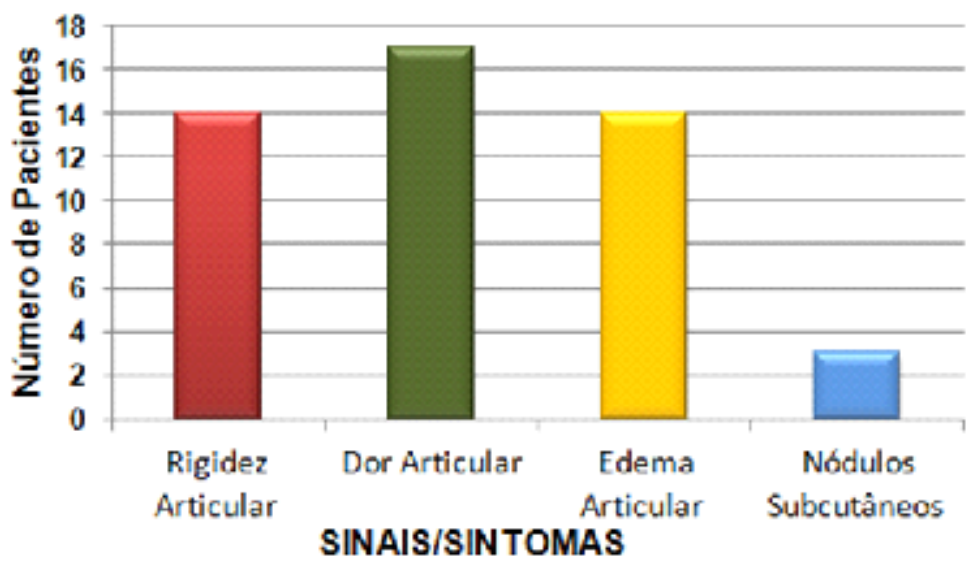

Gráfico 14. Sinais e Sintomas referidos pelos pacientes com AR atendidos no mbulatório e reumatologia SCMG-GO $(n=19)$

ocular, neuropatia periférica, síndrome de Sjögren, estas foram também bastante relatadas em estudos epidemiológicos nacionais. ${ }^{4,6}$

Os principais sinais e sintomas referidos pelos pacientes foram dor articular (89\%), rigidez matinal articular (73\%) e edema articular (73\%). Ressalta-se que a rigidez matinal e o edema tecidual apresentam alto valor prognóstico e fazem parte dos critérios para classificação da AR segundo o Colégio Americano de Reumatologia, o qual também destaca que constituem marcadores de prognóstico na AR, o número de articulações envolvidas, a presença de manifestações extra-articulares, anormalidades radiológicas, positividade do fator reumatóide e resultado desfavorável nos questionários de qualidade de vida. Em relação à impressão clínica geral da doença, neste estudo, uma minoria (22\%) foi classificada como grave, baseada nos critérios de prognóstico na AR, os indicadores clínicos (número de articulações envolvidas, grau de envolvimento extra-articular), os laboratoriais (altos níveis de PCR e VHS, altos títulos de FR) e os indicadores radiológicos (estreitamento do espaço articular e erosões ósseas). ${ }^{8,10}$

Laboratorialmente o fator reumatóide (FR) apresentou-se positivo em $68 \%$ dos pacientes e destes $26 \%$ eram positivos para autoanticorpos antinucleares (FAN). A AR envolve a produção de autoanticorpos onde o fator reumatóide (FR) está presente em $70 \%$ a $90 \%$ destes pacientes. No entanto, o FR tem especificidade de $59 \%$ a $65 \%$ podendo ser encontrado em outras condições clínicas (Lúpus Erite- 
matoso Sistêmico, Síndrome de Sjögren, Tireoidite autoimune, Hepatite Crônica e outras doenças). Apesar de não ser um marcador para diagnóstico, tem grande importância em relação ao prognóstico, pois pacientes com títulos altos e persistentes do mesmo têm maior gravidade e pior prognóstico. ${ }^{11,12,13}$

Outro marcador diagnóstico não solicitado e/ou relatado nos prontuários dos pacientes do hospital universitário (SCMG), neste estudo, foi o anticorpo antipeptídeo citrulinado cíclico (anti-CCP) o qual tem sido muito estudado quanto à sua importância como marcador para diagnóstico e com grande valor preditivo para o prognóstico na Artrite Reumatóide. Estudos correlacionam o anti-CCP com a progressão erosiva óssea da doença sugerindo que pacientes com altos títulos de anti-CCP possam ter doença mais agressiva e, portanto, pior prognóstico. $\mathrm{O}$ anti-CCP pode ser detectado em $25 \%$ dos indivíduos em 1,5 a 9 anos do aparecimento dos primeiros sintomas e no ano que antecede os sintomas sua sensibilidade aumenta para $52 \%$ indicando a eficiência desses testes em predizer o desenvolvimento futuro da Artrite Reumatóide. ${ }^{11,14,15}$

O diagnóstico diferencial da artrite reumatóide inclui outras poliartrites, a osteoartrose, a fibromialgia, algumas doenças sistêmicas autoimunes e outras doenças sistêmicas que podem apresentar dores articulares. Algumas doenças sistêmicas podem apresentar dores articulares como parte de seu quadro clínico, incluindo a endocardite bacteriana, o eritema nodoso, o hipotireoidismo, a anemia falciforme, a febre reumática, a febre familiar do mediterrâneo, entre outras. $\mathrm{Na}$ grande maioria destas doenças as dores articulares são de curta duração, não evoluem para deformidades articulares e geralmente fazem parte de uma ampla constelação de sintomas e sinais que não estão presentes na AR.18 Quanto as co-morbidades associadas à AR observou-se que 69\% dos pacientes apresentavam acometimento osteoarticular, como a osteoporose, osteoartrose, gota e síndrome da compactação, além de outras doenças crônicas comuns na população brasileira, tais como: hipertensão arterial sistêmica, diabetes mellitus, obesidade, hipotireoidismo, sendo compatíveis com alguns estudos brasileiros. ${ }^{8}$

Apenas $10 \%$ dos pacientes com AR do ambulatório de reumatologia do hospital escola SCMG realizavam tratamento fisioterapêutico e o fármaco (Droga Anti-Reumática Modificadora do Curso da Doença - DMARD) mais utilizado como tratamento medicamentoso da Artrite Reumatóide foi o metotrexato (dose média de $10 \mathrm{mg} / \mathrm{semana}$ ). Os DMARDs são medicamentos utilizados para modificar a história natural da AR. A intenção do médico, ao prescrever estas drogas, é evitar novos surtos de inflamação, induzindo e mantendo a remissão da doença. Para ser designado DMARD, o medicamento deve alterar o curso da AR ao menos por um ano, com evidências de melhora da capacidade funcional, diminuição da sinovite e retardo ou prevenção de dano articular estrutural observado ao exame radiológico. Segundo Louzada-Junior e colaboradores (2007) 23\% dos pacientes com AR do estado de São Paulo freqüentavam programas de reabilitação, principalmente fisioterapia. E confirma que o metotrexato ainda é a droga mais adotada no tratamento da Artrite Reumatóide nos serviços, $89 \%$ a utilizam ou já utilizaram, associado ou não a DMARDs. Estas são associadas a outros medicamentos, como, os analgésicos, antiinflamatórios e corticosteróides no combate dos sinais e sintomas da doença. A precocidade do diagnóstico, o início imediato das DMARDs e o controle rigoroso da atividade inflamatória podem melhorar a qualidade de vida do paciente e aumentar a sobrevida do mesmo. Atualmente, sabe-se que pacientes que participam de programas de exercícios fisioterapêuticos regulares melhoram a flexibilidade, a força, o estado cardiovascular e o funcionamento físico sem exacerbar a artrite ou aumentar os sintomas articulares. , $^{6,16,17}$

$\mathrm{O}$ perfil dos pacientes portadores de AR atendidos no ambulatório de reumatologia deste hospital universitário seria mulher (79\%), com idade variando entre 40 e 70 anos (73\%), 16\% apresentando história familiar de AR, história de início de doença há menos de dez anos (42\%), queixando-se de dor articular (89\%), em uso de analgésico ou antiinflamatório (89\%), em tratamento com metotrexato (79\%). Quase a metade dos pacientes (42\%) apresentava alterações articulares (radiológicas) já na primeira consulta com o reumatologista e mais da metade $(68 \%)$ apresentava alguma co-morbidade associada à doença. Cerca de um quarto desses pacientes apresentava manifestações extra-articulares $(21 \%)$, sendo o fator reumatóide sérico positivo em $68 \%$ dos pacientes. Esse perfil de paciente não difere muito do que é relatado em populações de outras regiões do Brasil e de outros países. $3,4,6,7$

\section{Conclusão}

O presente estudo se propôs a coletar dados epidemiológicos sobre as características demográfi- 
cas e clínicas de pacientes com Artrite Reumatóide atendidos no ambulatório acadêmico do Hospital Santa Casa de Misericórdia, localizado em Goiânia-GO. Apesar de ser uma análise descritiva retrospectiva com uma amostra pequena de pacientes $(n=19)$, este estudo proporcionou uma visão parcial do perfil epidemiológico da AR, levantando aspectos importantes relacionados à identificação, triagem dos pacientes, os critérios diagnósticos utilizados, tratamentos empregados, a pequena utilização de marcadores diagnósticos da doença e ainda foi possível detectar deficiências de informações nos prontuários médicos deste hospital universitário. Como devolutiva da academia (Departamento de Medicina da PUC Goiás) para o hospital escola de medicina, os autores deste trabalho, sob a orientação de um médico reumatologista, elaboraram uma ficha de evolução específica para o ambulatório de reumatologia que foi anexada aos prontuários deste setor ambulatorial, na tentativa de melhorar a qualidade e disponibilidade das informações médicas neste setor e, ainda foi sugerido a implementação de um sistema de prontuários eletrônicos à diretoria técnicaadministrativa do Hospital Escola Santa Casa de Misericórdia de Goiânia-GO, por meio do encaminhamento de um ofício e a criação de um programa de reabilitação física para atender pacientes reumáticos que necessitam de fisioterapia. Sabe-se que o centro-oeste é uma região muito carente de estudos epidemiológicos sobre doenças autoimunes reumáticas e, diante disso, percebe-se que falta despertar o interesse de profissionais da área de saúde para a realização de estudos abordando as doenças autoimunes reumáticas. Esperamos que esses dados epidemiológicos parciais possam auxiliar na caracterização do perfil dos pacientes com artrite reumatóide em Goiânia-GO, especialmente daqueles atendidos pelo Sistema Único de Saúde (SUS) da região centro-oeste. Também desejamos que os demais acadêmicos do curso de medicina da PUC Goiás, inseridos neste hospital universitário tenham uma visão crítico-reflexiva sobre os aspectos positivos e negativos dos serviços de saúde prestados à comunidade através desta unidade filantrópica de saúde com vistas a melhorar a qualidade do atendimento e tratamento dos pacientes.

\section{Agradecimentos}

Ao Comitê de Ética em Pesquisa do Hospital Universitário Santa Casa de Misericórdia de Goiânia pela concessão do estudo. Ao Departamento de Medicina da PUC Goiás e ao orientador do nosso TCC Prof. MSc. Hermínio Sobrinho.

\begin{abstract}
Objective: to perform an analysis of the main demographic and clinical features of patients with rheumatoid arthritis (RA), followed in outpatient in a Academic Medical Hospital in Goiania, Goias, Brazil. Methodology: This is an epidemiological study, retrospective, a set of patients with rheumatoid arthritis accompanied by a university hospital in the central-western Brazil. We reviewed medical records of 500 patients treated at the ambulatory of rheumatology in the period from 2011 to 2012. Data from medical records were transcribed into a document protocol standardized and validated by a physician rheumatologist. Results: From the 500 patients analyzed, 43\% had systemic rheumatic diseases, osteoarticular diseases $36 \%$, spondyloarthritides $10 \%$ and $11 \%$ other diseases. Of this total, $5 \%$ were patients with RA. The relationship between the female/male ratio was of $4: 1$, the mean age of patients was $50 \pm 13.3$ years, mean disease duration of $9.7 \pm 5,6$ years, $16 \%$ had a family history of RA, $42 \%$ presented radiological alterations at first visit and $69 \%$ presented co-morbidities. The main signs and symptoms reported by patients were joint pain (89\%), morning stiffness (73\%) and joint swelling (73\%). The extra-articular manifestations occurred in $21 \%$, rheumatoid factor was positive in $68 \%$ of patients and $26 \%$ of $\mathrm{RA}$ patients were positive for antinuclear autoantibodies. Only $10 \%$ of patients underwent physiotherapy treatment and more drug used as treatment of RA was the Methotrexate. Conclusion: This study provided a partial view of the epidemiological profile of the RA patients at a academic medical hospital in Goiania, Goias. There was a small use of diagnostic markers of the disease. Therefore, we propose a feedback to the rheumatology clinic in an attempt to improve the quality and availability of medical information in the records of this university hospital's outpatient at Pontificia Catholic University of Goias.
\end{abstract}

Keywords: Arthritis, Rheumatoid. Hospitals, Teaching. Epidemiological Profile. Goiania-GO. 


\section{REFERÊNCIAS BIBLIOGRÁFICAS}

1. Lee DM, Weinblatt ME. Rheumatoid arthritis. Lancet $2001 ; 358$ : 903-11.

2. Alarcón GS. Epidemiology of rheumatoid arthritis. Rheum Dis Clin North Am 1995; 21: 589-604.

3. Silman AJ, Pearson JE. Epidemiology and genetics of rheumatoid arthritis. Arthritis Res 2002; 4: 265-72.

4. Marques-Neto JF, Goncalves ET, Langen LFOB, Cunha MFL, Radominski S, Oliveira SM et al. Multicentric study of the prevalence of adult rheumatoid arthritis in Brazilian population samples. Rev Bras Reumatol 1993; 33: 169-73.

5. Schoels M, Wong J, Scott DL, Zink A, Richards P, Landewe R et al. Economic aspects of treatment options in rheumatoid arthritis: a systematic literature review informing the EULAR recommendations for the management of rheumatoid arthritis. Ann Rheum Dis. 2010; 69: 995-1003.

6. Louzada-Junior P, Souza BDB, Toledo RA, Ciconelli RM. Descriptive analysis of the demographical and clinical characteristics of the patients with rheumatoid arthritis in the State of Sao Paulo, Brazil. Rev Bras Reumatol. 2007; 47: 84-90.

7. Da Mota LM, Laurindo IM, dos Santos Neto LL. Demographic and clinical characteristics of a cohort of patients with early rheumatoid arthritis. Rev Bras Reumatol. 2010; 50: 235-48.

8. Mota LMH, Cruz BA, Brenol CV, Pereira IA, Fronza LST, Bertolo $\mathrm{MB}$ et al. Consenso da Sociedade Brasileira de Reumatologia 2011 para o diagnóstico e avaliação inicial da artrite reumatóide. Rev Bras Reumatol. 2011; 51: 207-19.

9. Lima-Costa MF, Barreto SM. Tipos de estudos epidemiológicos: conceitos básicos e aplicações na área do envelhecimento. Rev Epidem e Serv Saude. 2003; 3: 189 -201p.
10. Guidelines for the management of rheumatoid arthritis. American College of Rheumatology Ad Hoc Committee on Clinical Guidelines. Arthritis Rheum. 1996; 39:713-22.

11. Schellekens $G A$, Visser $H$, de Jong $B A$, van den Hoogen $F H$, Hazes JM, Breedveld FC et al. The diagnostic properties of rheumatoid arthritis antibodies recognizing a cyclic citrullinated peptide. Arthritis Rheum. 2000; 43: 155-63.

12. Teixeira RCA, Júnior AG, De Martino MC, Martins LC, Lopes AC, Tufik S. Marcadores de ativação endotelial e autoanticorpos na artrite reumatoide. Rev Bras Reumatol. 2007; 47: 411-17.

13. Lipsky PE. Artrite Reumatóide In: HARISSON. Medicina Inerna. V. 2, $14^{\mathrm{a}}$ ed. Rio de Janeiro: Editora McGraw-Hill Interamericana do Brasil LTDA; 1998.

14. Van Venroij WJ, Pruijn GJ. Citrullination: a small change for protein with great consequences for rheumatoid arthritis. Arthritis Res. 2000; 2: 249-51.

15. Nakamura RM. Progress in the use of biochemical and biological markers for evolution of rheumatoid arthritis. J Clin Lab Anal. 2000; 14: 305-13.

16. Albers JMC, Paimela L, Kurki P, Eberhardt KB, Emery P, van't Hof HM, et al. Treatment strategy, disease activity, and outcome in four cohorts of patients with early rheumatoid artrhitis. Ann Rheum Dis. 2001; 60: 453-8.

17. Klareskog L, Catrina AI, Paget S. Rheumatoid arthritis. Lancet. 2009; 373:659-72.

18. Harris ED, JR. Clinical features of rheumatoid arthritis. In: Kelley WN, Harris JED, Ruddy S, et al (eds). Textbook of Rheumatology, 4th ed. Philadelphia: WB Saunders 1993; $p$. 874-911. 